\title{
Simulator as a tool of training to modern equipment management
}

\author{
Gulnara Ahmedyanova ${ }^{1, *}$ \\ ${ }^{1}$ Orenburg state university, Orenburg, Russia
}

\begin{abstract}
In the work, the learning process with the use of a simulator was studied. In this case, both the design and algorithmic content of the simulator, as well as the trainee, must pass their part of the path, only in this case the result of learning is maximized. Theoretically, it is shown that the effectiveness of simulator training is primarily a function of the cognitive-operational and professional-personal aspects of the trainee's competence. The experiment confirmed that, despite the differences above the indicated qualities, the result can be estimated as the sum of their estimates.
\end{abstract}

\section{Introduction}

Modern technology is characterized by the tendency of continuous complication and increase in the speed of the processes occurring in it [1]. This, in turn, requires highly skilled professionals to manage it. The latter can not be achieved without sufficient practice for real management in various situations.

Of course, it is best to gain this practical experience on real equipment, but this is not always possible and often unsafe. In such conditions, the quasi-professional activity of the student is necessary, as the most appropriate tool for developing the necessary skills. Quasi-professional activity is understood here as maximally close to reality, so the best way to implement it is training on the simulator. An example is: training an astronaut to dock with an international space station, training a pilot, training a driver, training a surgeon, etc..

\section{Theory}

Trainers in the modern sense have appeared, when there was a necessity of mass preparation of experts for work or on the same type of equipment, or with similar working actions, and also for military needs. Recently, in connection with the rapid computerization of the world community, with the creation of sophisticated technology, the exploitation of which is associated with a risk to the lives of not only one person, but also of mankind as a whole, a whole industry has emerged-simulator technologies. Simulator technologies are complex compound, simulation and modeling systems, computer programs and physical models, special techniques designed to prepare the person for making quality and quick decisions. In modern simulators and in training and education programs based on them, the principles of development of practical skills with simultaneous theoretical preparation are laid [2].
Simulator technologies have emerged and developed most in those areas where errors in training on real objects can lead to extreme consequences, and their elimination to greater financial costs[3-6].

The simulator in the general case is a software and hardware complex. The dynamic response of the simulator should be as close as possible to the response of systems and components of the real object, for which virtual reality is widely used in simulators [7-12].

On the other hand, the simulator is an important representative of laboratory equipment. Unlike any other laboratory equipment, it is possible to learn exactly professional activity, rather than develop research skills. Under the simulator is usually understood as a training device for training, that is, for developing skills for the management of any machine or any mechanism. In another interpretation, the simulator is a mechanical, software, electric or combined training device that imitates artificially various loads or circumstances (situation). Simulator can be training (imitation) or sports.

To ensure its functions in accordance with the requirements of the principle of system accordance [13], the simulator must have an extended interface with trainees. When creating an interface, it is necessary to take into account the psychological characteristics of a person, for example, his sharp reaction to red, while the green color calms him, the information in the right side of the field of view draws attention faster and so on.

A part of the path must be trainee. When working on a simulator, it requires, apart from specialized knowledge: concentration, attentiveness, quick response, resourcefulness. Therefore, before working on the simulator, one should study a certain theoretical course.

Figure 1 shows a generalized scheme of the trainee's work on the simulator. Both the main components and the direct links between them are shown.

\footnotetext{
* Corresponding author: ahmedyanova@bk.ru
} 
The indicator of the effectiveness of training on the simulator in the general case can be represented in the

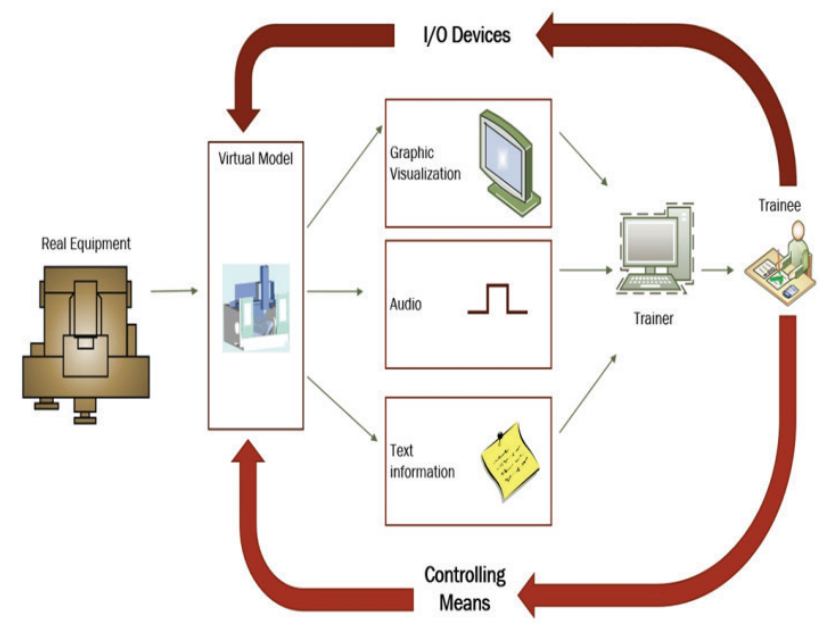

Fig.1. The scheme of the virtual simulator

form of some dependence on the totality of parameters of the simulator and the qualities of the trainees type [14]:

$$
F=F(N, M, S, P, V)
$$

where $\mathrm{N}$ is the set of typical situations for working out typical skills necessary for equipment management in standard situations;

$\mathrm{M}$ is the set of abnormal situations modeled on the simulator, (with the possibility of changing both their number and the intensity of the component faults of different classes, the types and parameters of the laws of distribution of time intervals between the time of receipt of various failures, etc.);

$\mathrm{S}$ - set of ergonomic factors;

$\mathrm{P}$ - the totality of theoretical knowledge about the managed equipment (cognitive-operational aspect of the trainee's competence [15]);

$\mathrm{V}$ - set of psychological qualities of the trainee, influencing the speed and correctness of making managerial decisions (professional-personal aspect of the trainee's competence [15]);

The first three sets N, M, S reflect the impact on the results of the learning process of the technical indicators of the simulator itself, while the last two sets reflect the influence of the personality traits of the trainee. More clearly in the linear expansion, the structure of the efficiency index can be expressed by the formula:

$$
\begin{gathered}
Q=\frac{\partial F}{\partial N} \Delta N+\frac{\partial F}{\partial M} \Delta M+\frac{\partial F}{\partial S} \Delta S+\frac{\partial F}{\partial P} \Delta P \\
+\frac{\partial F}{\partial V} \Delta V .
\end{gathered}
$$

here $\Delta N$ is the increment in the number of standard situations, $\Delta M$ is the increment in the number of contingencies, $\Delta S$ is the increment in the ergonomics level of the simulator, $\Delta P$ is the increment in the theoretical knowledge of the learner, $\Delta V$ is the increment in creative skills, and the derivatives in the formula reflect the influence of these increments on the overall efficiency.
On the other hand, the training time is also important. As with the passage of time only the competence of the trainee changes, we will evaluate the effectiveness of the reduced formula

$$
Q=\left(\frac{\partial F}{\partial P} \frac{\partial P}{\partial t}+\frac{\partial F}{\partial V} \frac{\partial V}{\partial t}\right) \Delta t .
$$

Now added derivatives additionally reflect the speed of mastering knowledge for students and the speed of learning management skills.

It follows that the evaluation of the effectiveness of the simulator can be reduced to a first approximation to assessing the quality of the organization of replenishment of knowledge and creative abilities and skills for students.

\section{Data and Methods}

In this study, EMCO virtual classroom training simulators were used to develop practical skills in the process of preparing and upgrading teachers, as well as for automated assessment of knowledge and skills for different levels of complexity of work on the milling machine.

\section{Methods or Model}

The simulators used in the study are a software and hardware complex interacting with the user through interactive dialogue means (mouse, keyboard), a control panel model with visualization of the state of the technological process (See Figure 2), equipment and mechanisms based on modern multimedia technologies for presenting information (text, graphics, sound ).

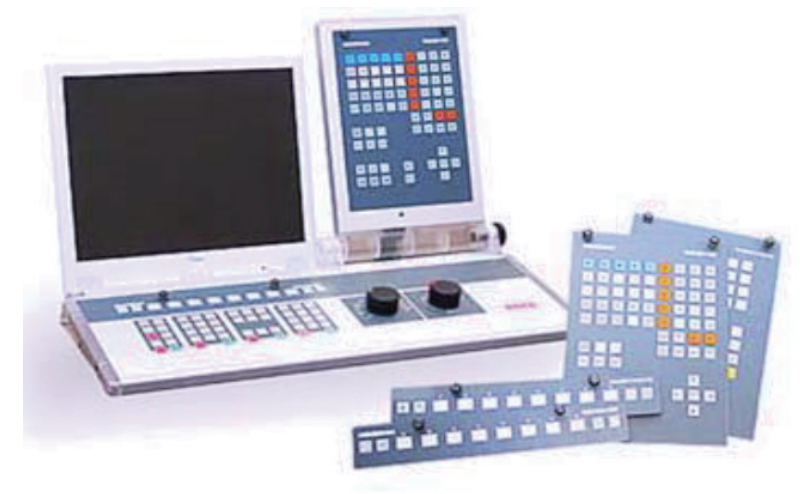

Fig. 2. Appearance of the simulator

The program interface ensures the operation of the complex provided that personnel who are not a qualified user of the PC work there. The training provides an imitation of the technological process applied to the production technology on the machine, provides a concise, easy-to-assimilate form of representation on the main and auxiliary information display. When working with simulators, basic didactic principles of cognitive activity are provided, access to reference and training material, training for developing practical work skills and control over the degree of mastering theoretical and 
practical knowledge. The software product contains methodological and didactic materials for training on the simulator in accordance with educational programs.

In the development of simulator software, the modular principles of program design were envisaged, providing the possibility of increasing and upgrading the functionality of simulators [16]. The simulator software functions both in stand-alone mode (on a separate local machine) and in a local network. When working in a local network, several (up to 10) training places of the simulator interact with the instructor's workplace.

1. Module "Device of equipment and control panel" is designed to study: - machine tool; - devices of control panels and the purpose of machine controls; arrangements for setting and resetting the machine; - ways to control the products; - types of product quality mismatch with an indication of the causes and methods of elimination.

2. In the module "Technological process" a virtual model of the machine and control panels is realized, interacting with which the trainee can perform all actions on the machine during the working shift: - selection of the tool in accordance with the current task; - start the machine; - execution of technological operations; stopping the machine; - quality control of the products; adjustment of the machine settings depending on the quality mismatch obtained (the type of nonconformity is selected randomly). The module can work in the training mode (prompts and explanations are given during operation), and in the knowledge testing mode (no prompts are given, the correctness of work on the machine is evaluated).

3. The module "Emergency situations" implements a demonstration and description of the main types of emergency situations, methods for their prevention and elimination of consequences in accordance with technological instructions and instructions on safety. For each emergency situation, the following is displayed: description of the emergency situation; - visual manifestations; - causes of occurrence; - ways of elimination.

4. The module "Testing the knowledge gained in the learning process" consists of a theoretical and practical part. At the end of each part, an assessment is made on a 5-point scale. The theoretical part is questions with the answers, questions are chosen randomly from the base of questions. A mechanism is provided for changing the base of questions with answers from the teacher's workplace. The practical part is the module "Technological process" in the mode of knowledge testing. Knowledge testing is possible on several levels of complexity. A list of difficulty levels and the correspondence of questions to different levels are set from the teacher's place.

The trainee can perform all the actions on the machine: - selecting the tool according to the current job; - start the machine; - execution of technological operations; stopping the machine; - quality control of the products; adjustment of the machine settings depending on the quality mismatch obtained (the type of nonconformity is selected randomly). The module can work in the training mode (prompts and explanations in process of work), and in the knowledge testing mode (no prompts are given, the correctness of work on the machine is evaluated). An illustration of the complexity of the material being studied can be the device of a keyboard of controlled equipment (see Figure 3)

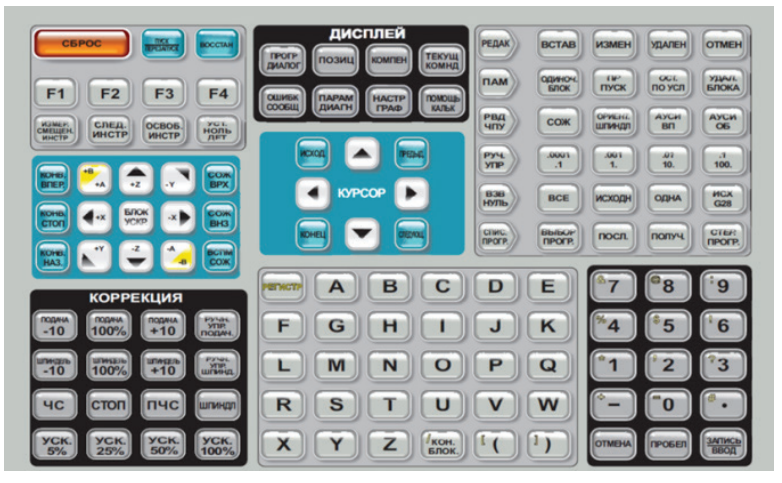

Fig. 3. General view of the console

\section{Results}

The assimilation of knowledge was estimated by the volumes of the acquired textual information, and the creative skills and skills by the number of mastered operations from which the typical actions were composed. The result of assimilation was estimated in points and reflected by the diagram in Figure 4

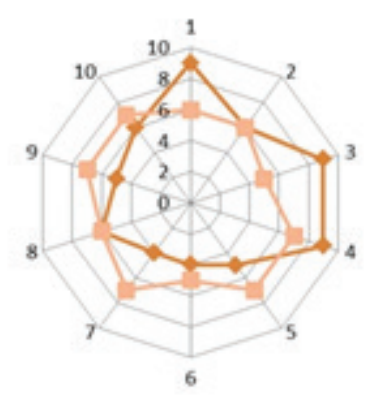

Fig.4. The pie chart of the abilities of students

The result of training as a sum of two aspects of educational activity is reflected by the histogram in Figure 5.

From the first diagram it follows that the cognitiveoperational and professional-personal aspects of students in the group differ significantly, but the best results of training were shown by students with a high level of both these aspects.

\section{Conclusions or discussion and implication}

Thus, the simulator is an effective tool of training to modern equipment management. Moreover, the design 


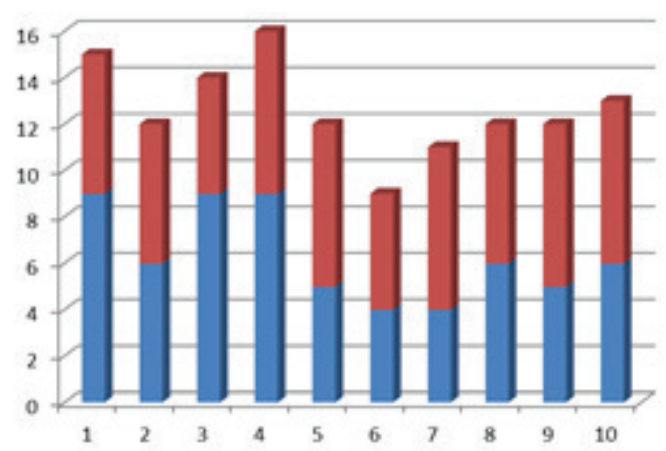

Fig.5. Training Effect Histogram

and algorithmic filling of the simulator, as well as the trainee, must pass their part of the path, only in this case the result is maximized.

Training is provided by a quasi-professional activity that simulates the control of the technological process with respect to the production technology on these machines, provides a concise, easy-to-digest form of representation on the main and auxiliary information display. When working with simulators, basic didactic principles of cognitive activity are provided, access to reference and training material, training for developing practical work skills and control over the degree of mastering theoretical and practical knowledge.

\section{Reference}

1. A.M. Pishchukhin, GF.Akhmedyanova, Automation and technological development are two aspects of the technical equipment of production, Bulletin of the Orenburg State University, 9(184), pp. 170-174 (2015)

2. A.V. Trukhin, Analysis of existing in Russia simulators and training systems. Access mode: https://ido.tsu.ru/other_res/pdf/1(29)_2008(3239).pdf

3. V.V. Belov, I.V. Obraztsov, Virtualization of physical processes in the theory and practice of building education. The theory and practice of increasing efficiency builds, Materials: Materials of $\mathrm{V}$ Vseros. Conf. Students, graduate students and young scientists. Penza: PGUAS, pp. 186-189 (2010)

4. A.P. Blinov, Virtual training simulator: effective and safe, Vocational education and the labor market $\mathbf{2}$ (2013)

5. A.O. Matlin, D.S. Osintsev, S.A. Fomenkov, Automated system "interactive virtual simulators", Proceedings of the V All-Russian Conference of Students, $\mathrm{PhD}$ students and young scientists "Microsoft Technology in the theory and practice of programming." Central region (Moscow: The University Book, p. 75-77, 2008)

6. L. Soyeon, P. Sangjoon, C. Kyoil and C. Choongho Kinematic Skeleton Based Control of a Virtual Simulator for Military Training, Symmetry 7(2), p. 1043-1060 (2015)
7. V.O. Afanasyev, A.G. Brovkin, A.N. Kornievsky, V.P. Podobedov, V.S. Semchenko, A.N. Tomilin, Research and develop-ment of the interactive observation system of the induced virtual environment (virtual presence systems), Cosmonautics and rocket science, 20 (2001)

8. A.O. Matlin, D.S. Osintsev, V.V. Get-mansky, S.A. Fomenkov, Interactive virtual medical simulators, Proceedings of the VI All-Russian Conference of 22 students, graduate students and young scientists "Microsoft Technologies in the theory and practice of programming." Central region (Moscow: Moscow. Aviation institute, p. 122-123, 2009)

9. B. Dalgarno, and M. J. W Lee, What are the Learning Affordances of $3 D$ Virtual Environments?, British Journal of Educational Technology, 41(1) (2010), 10-32

10. J. Moskaliuk, J. Bertram \& U. Cress, Training in virtual environments: putting theory into practice, Ergonomics 56(2), p. 195-204 (2013)

11. F. D. Rose, E. A.Attree, B. M. Brooks, D. M. Parslow, and P. R. Penn, Training in Virtual Environments: Transfer to Real World Tasks and Equivalence to Real Task Training, Ergonomics, 43(4), p. 494-511 (2000)

12. J. G. Tichon, Using Presence to Improve a Virtual Training Environment, CyberPsychology \& Behavior, 10(6), p.781-7. (2007)

13. A.M. Pishchukhin, Accordance of the components of the system and methods for achieving it, Bulletin of the Orenburg State University, 1, p. 87-90 (1999)

14. A.N. Privalov, Evaluation of the effectiveness of simulators with distributed data processing, Scientific bulletin 1(56) (2009)

15. G.F. Ahmedyanova, Features of development of cognitive-operational and professional-personal competencies of bachelors, Fundamental research, 220, pp. 4492-4495 (2015)

16. I.P. Norenkov, A.M. Zimin, Information technologies in education, (M.: Publishing House of the Moscow State Technical University. N.E. Bauman, 2004) 